\title{
Multitask CapsNet: An Imbalanced Data Deep Learning Method for Predicting Toxicants
}

\section{SUPPLEMENTARY DATA}

Yiwei Wang, ${ }^{\mathrm{a}, \mathrm{c}}$ Binyou Wang, ${ }^{\mathrm{b}}$ Jie Jiang, ${ }^{\mathrm{a}}$ Jianmin Guo, ${ }^{\mathrm{a}}$ Jia Lai, ${ }^{\mathrm{b}}$

\author{
Xiao-Yuan Lian*d, and Jianming $\mathrm{Wu}^{* \mathrm{c}}$
}

${ }^{a}$ School of Preclinical Medicine, Southwest Medical University, Luzhou 646000,

China.

${ }^{b}$ School of Pharmacy, Southwest Medical University, Luzhou 646000, China.

${ }^{c}$ Key Laboratory of Medical Electrophysiology, Ministry of Education of China, Medical Key Laboratory for Drug Discovery and Druggability Evaluation of Sichuan Province, Luzhou Key Laboratory of Activity Screening and Druggability Evaluation for Chinese Materia Medica, Luzhou 646000, China.

${ }^{d}$ School of Pharmacy, Zhejiang University, Hangzhou 310011, China.

\section{S1: Supplementary Parameters}

The contribution of each capsule $i$ in PrimaryCaps to each capsule $j$ in CapsNets is computed as follows:

$$
\widehat{u}_{j \mid i}=W_{i j} \cdot u_{i}
$$

Parameter $u_{i}$ is considered as the output of capsule $i$, and $\widehat{u}_{j \mid i}$ is a prediction vector representing a weighted contribution of each low-level capsule $i$ to high-level capsule $j$. $W_{i j}$ is a weighting matrix that needs to be learned in backward pass calculations. Based on the degree of conformation between the capsules in the layer bellow and the 
parent capsules, coupling coefficients $c_{i j}$ are calculated using the following softmax function:

$$
c_{i j}=\frac{\exp \left(b_{i j}\right)}{\sum_{k} \exp \left(b_{i k}\right)}
$$

where $b_{i j}$ represents the log probability of the coupling strength between capsule $i$ and capsule $j$. The value of $b_{i j}$ is initiated to 0 before the routing-by-agreement process. The value of $c_{i j}$ is then updated by an iterative dynamic routing process. If the coupling coefficient $c_{i j}$ between capsule $i$ and capsule $j$ is 1 , the coupling coefficient between capsule $i$ and other high-level capsules is 0 .

To determine the total input vector $s_{j}$ of each high-level capsule $j$, a weighted sum over all prediction vectors is defined as follows:

$$
s_{j}=\sum_{i} c_{i j} \widehat{u}_{j \mid i}
$$

The output vector $v_{j}$ of high-level capsule $j$ is then computed using a non-linear squashing function:

$$
v_{j}=\frac{\left\|s_{j}\right\|^{2}}{1+\left\|s_{j}\right\|^{2}} \frac{s_{j}}{\left\|s_{j}\right\|}
$$

The loss $L_{k}$ for each capsule $k$ in CapsNets is computed as follows:

$$
L_{k}=T_{k} \max \left(0, m^{+}-\left\|v_{k}\right\|\right)^{2}+\lambda\left(1-T_{k}\right) \max \left(0,\left\|v_{k}\right\|-m^{-}\right)^{2}
$$

where $T_{k}=1$ if the predicted value is equal to the real, value and $T_{k}=0$ otherwise. $\mathrm{m}^{+}$, $\mathrm{m}^{-}$, and $\lambda$ are hyper-parameters that must be predefined. We used $\mathrm{m}^{+}=0.9, \mathrm{~m}^{-}=0.1$, and $\lambda=0.5$, which have been shown to ensure the stability of the training process.

The RBMs used the following energy function as the loss function:

$$
E(v, h)=-\left(a^{T} \cdot v+b^{T} \cdot h+v^{T} \cdot \omega \cdot h\right)
$$

Where $v \in\{0,1\}^{n}$ of visible layers is used to input data and $h \in\{0,1\}^{n}$ of hidden layers represent feature encoding for information of data. Parameters $a, b$ and $w$ represent weighting matrix.

The "frequency of a fragment" enrichment factor in chemicals was defined as follows:

$$
\text { Frequency of a fragment }=\frac{N_{\text {fragment_P }} X N_{\text {total }}}{N_{\text {fragment_total }} \times N_{P}}
$$

Where $N_{\text {fragment }}$ is the number of positive compounds containing the fragment in each class; $N_{\text {total }}$ is the total number of compounds used in this study; $N_{\text {fragment_total }}$ the total number of compounds containing the fragment; and $N_{p}$, the number of positive compounds. 


\section{S2: Selection of the Optimal Architectures}

Table S1. Prediction Performance of Different Network Architectures of Multitask CapsNet Models on Training Data Set by Fivefold Cross-validation.

\begin{tabular}{|c|c|c|c|c|c|}
\hline $\begin{array}{c}\text { network } \\
\text { architecture }\end{array}$ & SE & SP & MCC & $\mathbf{Q}$ & ROC \\
\hline RBM & $50.2 \pm 11.2 \%$ & $97.3 \pm 2.3 \%$ & $0.533 \pm 0.133$ & $94.4 \pm 3.1 \%$ & $0.908 \pm 0.052$ \\
\hline $\mathbf{R B M + R B M}$ & $52.8 \pm 11.6 \%$ & $97.7 \pm 2.2 \%$ & $0.560 \pm 0.172$ & $95.3 \pm 2.2 \%$ & $0.920 \pm 0.035$ \\
\hline $\begin{array}{l}\mathrm{RBM}+\mathrm{RBM}+ \\
\mathrm{RBM}\end{array}$ & $48.2 \pm 12.0 \%$ & $98.0 \pm 1.1 \%$ & $0.532 \pm 0.132$ & $94.1 \pm 3.0 \%$ & $0.893 \pm 0.030$ \\
\hline $\begin{array}{l}\mathrm{RBM}+\mathrm{RBM}+ \\
\mathrm{RBM}+\mathrm{RBM}\end{array}$ & $49.3 \pm 11.4 \%$ & $96.8 \pm 2.0 \%$ & $0.518 \pm 0.142$ & $93.8 \pm 4.3 \%$ & $0.878 \pm 0.031$ \\
\hline $\mathrm{RBM}+\mathrm{RBM}+$ & & & & & \\
\hline $\begin{array}{l}\mathrm{RBM}+\mathrm{RBM}+ \\
\mathrm{RBM}\end{array}$ & $47.0 \pm 10.1 \%$ & $97.2 \pm 1.7 \%$ & $0.499 \pm 0.181$ & $94.0 \pm 4.2 \%$ & $0.881 \pm 0.034$ \\
\hline
\end{tabular}

Abbreviations: RBM: restricted Boltzmann machine, SE: sensitivity, SP: specificity, MCC: Matthe's correlation coefficient, Q: accuracy, ROC: receiver operating characteristic curve.

\section{S3: Performances of Five Multitask Models}

Table S2. Prediction Performance of Different Multitask Models and Single Task CapsNet on Training Dataset by Fivefold Cross-validation.

\begin{tabular}{ccccc}
\hline models & SE & SP & MCC & Q \\
\hline Multitask DBN & $25.8 \pm 18.1 \%$ & $99.1 \pm 0.9 \%$ & $0.404 \pm 0.141$ & $93.8 \pm 4.7 \%$ \\
Multitask CNN & $42.2 \pm 13.0 \%$ & $98.2 \pm 1.5 \%$ & $0.491 \pm 0.132$ & $94.1 \pm 3.5 \%$ \\
Multitask LSTM & $19.9 \pm 15.0 \%$ & $97.7 \pm 2.2 \%$ & $0.249 \pm 0.181$ & $93.4 \pm 4.2 \%$ \\
Multitask MLP & $41.3 \pm 18.4 \%$ & $99.4 \pm 2.5 \%$ & $0.419 \pm 0.133$ & $92.3 \pm 4.2 \%$ \\
Multitask CapsNet & $52.8 \pm 11.6 \%$ & $97.7 \pm 2.2 \%$ & $0.560 \pm 0.172$ & $95.3 \pm 4.2 \%$ \\
Single task CapsNet & $50.1 \pm 9.6 \%$ & $96.8 \pm 1.8 \%$ & $0.507 \pm 0.087$ & $93.2 \pm 3.5 \%$ \\
\hline
\end{tabular}


Table S3. Prediction Performance of All Multitask Models for the 12 Separate Targets on Leaderboard Data Set.

\begin{tabular}{|c|c|c|c|c|}
\hline task & model & SE & SP & $\mathbf{Q}$ \\
\hline & Multitask DBN & $52.5 \%$ & $98.4 \%$ & $93.0 \%$ \\
\hline & Multitask CNN & $71.9 \%$ & $98.7 \%$ & $95.6 \%$ \\
\hline \multirow[t]{5}{*}{ NR. AhR } & Multitask LSTM & $33.8 \%$ & $98.3 \%$ & $90.8 \%$ \\
\hline & Multitask MLP & $66.7 \%$ & $97.7 \%$ & $94.1 \%$ \\
\hline & Multitask CapsNet & $79.2 \%$ & $98.4 \%$ & $96.3 \%$ \\
\hline & Multitask DBN & $0.0 \%$ & $100.0 \%$ & $99.0 \%$ \\
\hline & Multitask CNN & $0.0 \%$ & $98.6 \%$ & $97.6 \%$ \\
\hline \multirow[t]{5}{*}{ NR. AR } & Multitask LSTM & $0.0 \%$ & $100.0 \%$ & $97.9 \%$ \\
\hline & Multitask MLP & $0.0 \%$ & $96.5 \%$ & $95.5 \%$ \\
\hline & Multitask CapsNet & $0.0 \%$ & $99.9 \%$ & $97.9 \%$ \\
\hline & Multitask DBN & $58.5 \%$ & $99.7 \%$ & $98.0 \%$ \\
\hline & Multitask CNN & $75.7 \%$ & $99.7 \%$ & $98.4 \%$ \\
\hline \multirow[t]{5}{*}{ NR.AR.LBD } & Multitask LSTM & $60.2 \%$ & $99.6 \%$ & $97.6 \%$ \\
\hline & Multitask MLP & $62.4 \%$ & $99.4 \%$ & $94.5 \%$ \\
\hline & Multitask CapsNet & $81.7 \%$ & $99.7 \%$ & $97.6 \%$ \\
\hline & Multitask DBN & $0.0 \%$ & $99.5 \%$ & $91.1 \%$ \\
\hline & Multitask CNN & $38.9 \%$ & $90.3 \%$ & $86.0 \%$ \\
\hline \multirow[t]{5}{*}{ NR. Aromatase } & Multitask LSTM & $0.0 \%$ & $100.0 \%$ & $91.6 \%$ \\
\hline & Multitask MLP & $16.7 \%$ & $91.3 \%$ & $85.0 \%$ \\
\hline & Multitask CapsNet & $38.9 \%$ & $96.9 \%$ & $92.1 \%$ \\
\hline & Multitask DBN & $11.1 \%$ & $96.2 \%$ & $87.5 \%$ \\
\hline & Multitask CNN & $11.1 \%$ & $92.0 \%$ & $83.8 \%$ \\
\hline \multirow[t]{3}{*}{ NR. ER } & Multitask LSTM & $7.4 \%$ & $99.3 \%$ & $89.4 \%$ \\
\hline & Multitask MLP & $22.2 \%$ & $96.30 \%$ & $76.60 \%$ \\
\hline & Multitask CapsNet & $44.4 \%$ & $98.6 \%$ & $91.7 \%$ \\
\hline NR.ER.LBD & Multitask DBN & $10.0 \%$ & $97.8 \%$ & $94.8 \%$ \\
\hline
\end{tabular}




\begin{tabular}{|c|c|c|c|c|}
\hline & Multitask CNN & $20.0 \%$ & $92.8 \%$ & $90.2 \%$ \\
\hline & Multitask LSTM & $20.0 \%$ & $99.3 \%$ & $96.5 \%$ \\
\hline & Multitask MLP & $20.0 \%$ & $96.30 \%$ & $93.7 \%$ \\
\hline & Multitask CapsNet & $40.0 \%$ & $98.60 \%$ & $96.5 \%$ \\
\hline & Multitask DBN & $0.00 \%$ & $100.0 \%$ & $94.4 \%$ \\
\hline & Multitask CNN & $20.0 \%$ & $92.0 \%$ & $89.5 \%$ \\
\hline NR.PPAR.gamma & Multitask LSTM & $0.00 \%$ & $100.0 \%$ & $94.4 \%$ \\
\hline & Multitask MLP & $20.0 \%$ & $96.4 \%$ & $93.7 \%$ \\
\hline & Multitask CapsNet & $53.3 \%$ & $99.2 \%$ & $96.6 \%$ \\
\hline & Multitask DBN & $26.0 \%$ & $92.0 \%$ & $84.5 \%$ \\
\hline & Multitask CNN & $58.3 \%$ & $73.7 \%$ & $70.5 \%$ \\
\hline SR. ARE & Multitask LSTM & $14.6 \%$ & $97.8 \%$ & $80.8 \%$ \\
\hline & Multitask MLP & $50.0 \%$ & $77.4 \%$ & $71.7 \%$ \\
\hline & Multitask CapsNet & $62.5 \%$ & $93.0 \%$ & $86.8 \%$ \\
\hline & Multitask DBN & $0.0 \%$ & $100.0 \%$ & $90.8 \%$ \\
\hline & Multitask CNN & $4.0 \%$ & $4.0 \%$ & $88.9 \%$ \\
\hline SR. ATAD5 & Multitask LSTM & $0.0 \%$ & $100.0 \%$ & $90.8 \%$ \\
\hline & Multitask MLP & $8.0 \%$ & $98.3 \%$ & $90.0 \%$ \\
\hline & Multitask CapsNet & $32.0 \%$ & $98.8 \%$ & $92.7 \%$ \\
\hline & Multitask DBN & $10.0 \%$ & $100.0 \%$ & $96.6 \%$ \\
\hline & Multitask CNN & $30.0 \%$ & $93.7 \%$ & $91.4 \%$ \\
\hline SR. HSE & Multitask LSTM & $0.0 \%$ & $100.0 \%$ & $96.3 \%$ \\
\hline & Multitask MLP & $20.0 \%$ & $93.7 \%$ & $91.0 \%$ \\
\hline & Multitask CapsNet & $60.0 \%$ & $92.6 \%$ & $91.4 \%$ \\
\hline & Multitask DBN & $0.0 \%$ & $100.0 \%$ & $84.0 \%$ \\
\hline & Multitask CNN & $63.4 \%$ & $96.0 \%$ & $74.3 \%$ \\
\hline SR. MMP & Multitask LSTM & $37.4 \%$ & $96.8 \%$ & $81.1 \%$ \\
\hline & Multitask MLP & $58.1 \%$ & $93.7 \%$ & $73.9 \%$ \\
\hline & Multitask CapsNet & $61.4 \%$ & $98.5 \%$ & $88.2 \%$ \\
\hline
\end{tabular}




\begin{tabular}{ccccc} 
& Multitask DBN & $14.3 \%$ & $97.9 \%$ & $\mathbf{8 9 . 2 \%}$ \\
Multitask CNN & $53.5 \%$ & $85.0 \%$ & $81.7 \%$ \\
SR. p53 & Multitask LSTM & $0.0 \%$ & $100.0 \%$ & $89.6 \%$ \\
& Multitask MLP & $17.8 \%$ & $90.8 \%$ & $83.2 \%$ \\
& Multitask CapsNet & $71.4 \%$ & $90.5 \%$ & $88.5 \%$ \\
\hline
\end{tabular}

\section{S4: Comparison with DeepTox}

Table S4. AUC Results of Multitask CapsNet and Multitask DNN Models of DeepTox in the Literature on Leaderboard Data Set.

\begin{tabular}{ccc}
\hline task & DNN & CapsNet \\
NR. AhR & 0.841 & $\mathbf{0 . 8 9 0}$ \\
NR. AR & 0.346 & $\mathbf{0 . 4 9 5}$ \\
NR.AR.LBD & $\mathbf{0 . 9 2 9}$ & 0.820 \\
NR. Aromatase & 0.792 & $\mathbf{0 . 8 0 6}$ \\
NR. ER & 0.695 & $\mathbf{0 . 8 1 2}$ \\
NR.ER.LBD & 0.727 & $\mathbf{0 . 8 2 0}$ \\
NR.PPAR.gamma & 0.710 & $\mathbf{0 . 8 4 9}$ \\
SR. ARE & 0.802 & $\mathbf{0 . 8 5 6}$ \\
SR. ATAD5 & 0.796 & $\mathbf{0 . 8 8 3}$ \\
SR. HSE & 0.810 & $\mathbf{0 . 8 9 9}$ \\
SR. MMP & 0.849 & $\mathbf{0 . 9 0 3}$ \\
SR. p53 & 0.749 & $\mathbf{0 . 8 5 9}$ \\
\hline
\end{tabular}


Table S5. AUC Results of Multitask CapsNet and Various Models of DeepTox in the Literature on Test Set.

\begin{tabular}{ccccc}
\hline task & DNN & SVM & RF & CapsNet \\
\hline NR. AhR & 0.923 & 0.919 & 0.917 & $\mathbf{0 . 9 2 6}$ \\
NR. AR & 0.778 & 0.822 & 0.776 & $\mathbf{0 . 8 5 7}$ \\
NR.AR.LBD & $\mathbf{0 . 8 2 5}$ & 0.748 & 0.812 & 0.774 \\
NR. Aromatase & 0.804 & 0.819 & 0.806 & $\mathbf{0 . 8 3 1}$ \\
NR. ER & 0.791 & 0.799 & 0.770 & $\mathbf{0 . 8 3 5}$ \\
NR.ER.LBD & 0.811 & 0.798 & 0.746 & $\mathbf{0 . 8 4 6}$ \\
NR.PPAR.gamma & 0.856 & 0.827 & 0.805 & $\mathbf{0 . 9 2 0}$ \\
SR. ARE & 0.829 & 0.818 & 0.810 & $\mathbf{0 . 8 5 7}$ \\
SR. ATAD5 & 0.775 & 0.781 & 0.786 & $\mathbf{0 . 8 2 6}$ \\
SR. HSE & 0.863 & 0.848 & 0.826 & $\mathbf{0 . 8 8 4}$ \\
SR. MMP & 0.930 & $\mathbf{0 . 9 4 6}$ & 0.945 & 0.930 \\
SR. p53 & 0.860 & 0.854 & 0.835 & $\mathbf{0 . 8 9 2}$ \\
\hline
\end{tabular}




\section{S5: Performance on Balanced Datasets}

Table S6. The Number of Positive and Negative Compounds in the Original Unbalanced and New Balanced Training Sets from Tox 21 Data Set.

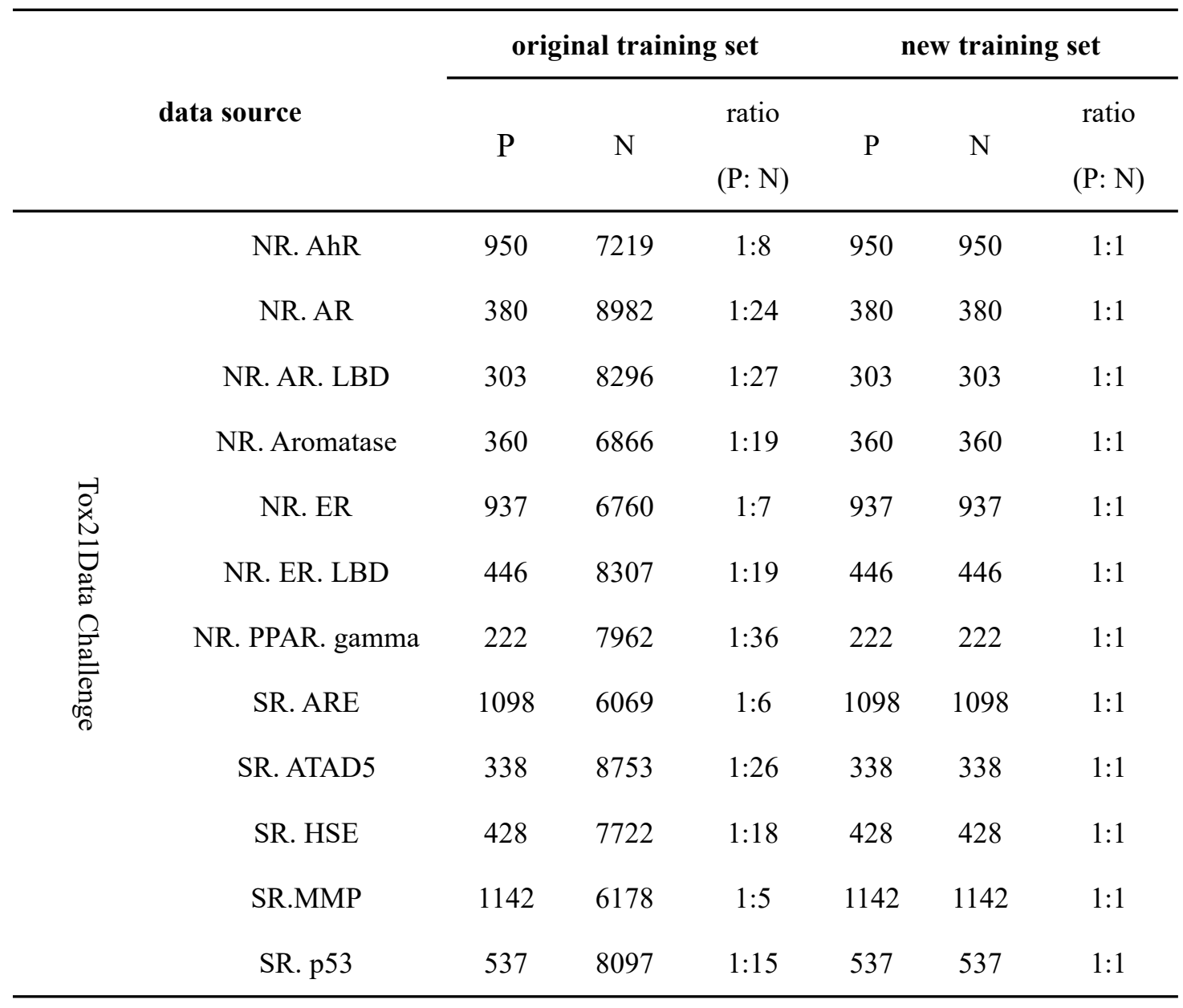


Table S7. Prediction Performance of Multitask CapsNet and Multitask DBN Models on Leaderboard Dataset for the 12 Separate Targets Training on Balanced Data Sets.

\begin{tabular}{|c|c|c|c|c|}
\hline task & model & SE & SP & $\mathbf{Q}$ \\
\hline \multirow{2}{*}{ NR. AhR } & Multitask DBN & $83.9 \%$ & $63.9 \%$ & $66.4 \%$ \\
\hline & Multitask CapsNet & $80.6 \%$ & $73.4 \%$ & $74.3 \%$ \\
\hline \multirow{2}{*}{ NR. AR } & Multitask DBN & $33.3 \%$ & $73.0 \%$ & $72.6 \%$ \\
\hline & Multitask CapsNet & $66.7 \%$ & $73.4 \%$ & $74.1 \%$ \\
\hline \multirow{2}{*}{ NR.AR.LBD } & Multitask DBN & $50.0 \%$ & $77.1 \%$ & $76.7 \%$ \\
\hline & Multitask CapsNet & $50.0 \%$ & $90.0 \%$ & $89.3 \%$ \\
\hline \multirow{2}{*}{ NR. Aromatase } & Multitask DBN & $38.9 \%$ & $62.2 \%$ & $60.3 \%$ \\
\hline & Multitask CapsNet & $38.9 \%$ & $77.6 \%$ & $74.3 \%$ \\
\hline \multirow{2}{*}{ NR. ER } & Multitask DBN & $44.4 \%$ & $63.4 \%$ & $61.5 \%$ \\
\hline & Multitask CapsNet & $22.2 \%$ & $83.6 \%$ & $77.4 \%$ \\
\hline \multirow{2}{*}{ NR.ER.LBD } & Multitask DBN & $70.0 \%$ & $66.4 \%$ & $66.6 \%$ \\
\hline & Multitask CapsNet & $40.0 \%$ & $87.0 \%$ & $85.4 \%$ \\
\hline \multirow{2}{*}{ NR.PPAR.gamma } & Multitask DBN & $60.0 \%$ & $74.6 \%$ & $73.8 \%$ \\
\hline & Multitask CapsNet & $66.7 \%$ & $84.9 \%$ & $88.8 \%$ \\
\hline \multirow{2}{*}{ SR. ARE } & Multitask DBN & $25.0 \%$ & $87.6 \%$ & $74.8 \%$ \\
\hline & Multitask CapsNet & $62.5 \%$ & $82.3 \%$ & $78.2 \%$ \\
\hline \multirow{2}{*}{ SR. ATAD5 } & Multitask DBN & $64.0 \%$ & $59.9 \%$ & $60.3 \%$ \\
\hline & Multitask CapsNet & $80.0 \%$ & $72.5 \%$ & $73.2 \%$ \\
\hline \multirow{2}{*}{ SR. HSE } & Multitask DBN & $80.0 \%$ & $61.1 \%$ & $61.8 \%$ \\
\hline & Multitask CapsNet & $80.0 \%$ & $84.0 \%$ & $83.9 \%$ \\
\hline \multirow{2}{*}{ SR. MMP } & Multitask DBN & $44.7 \%$ & $81.5 \%$ & $75.6 \%$ \\
\hline & Multitask CapsNet & $76.3 \%$ & $84.0 \%$ & $82.8 \%$ \\
\hline \multirow{2}{*}{ SR. p53 } & Multitask DBN & $67.9 \%$ & $65.6 \%$ & $65.8 \%$ \\
\hline & Multitask CapsNet & $64.3 \%$ & $68.5 \%$ & $68.0 \%$ \\
\hline
\end{tabular}




\section{S6: Algorithm and Training Procedure}

Table S8. Algorithm and Training Procedure of Multitask CapsNet Model.

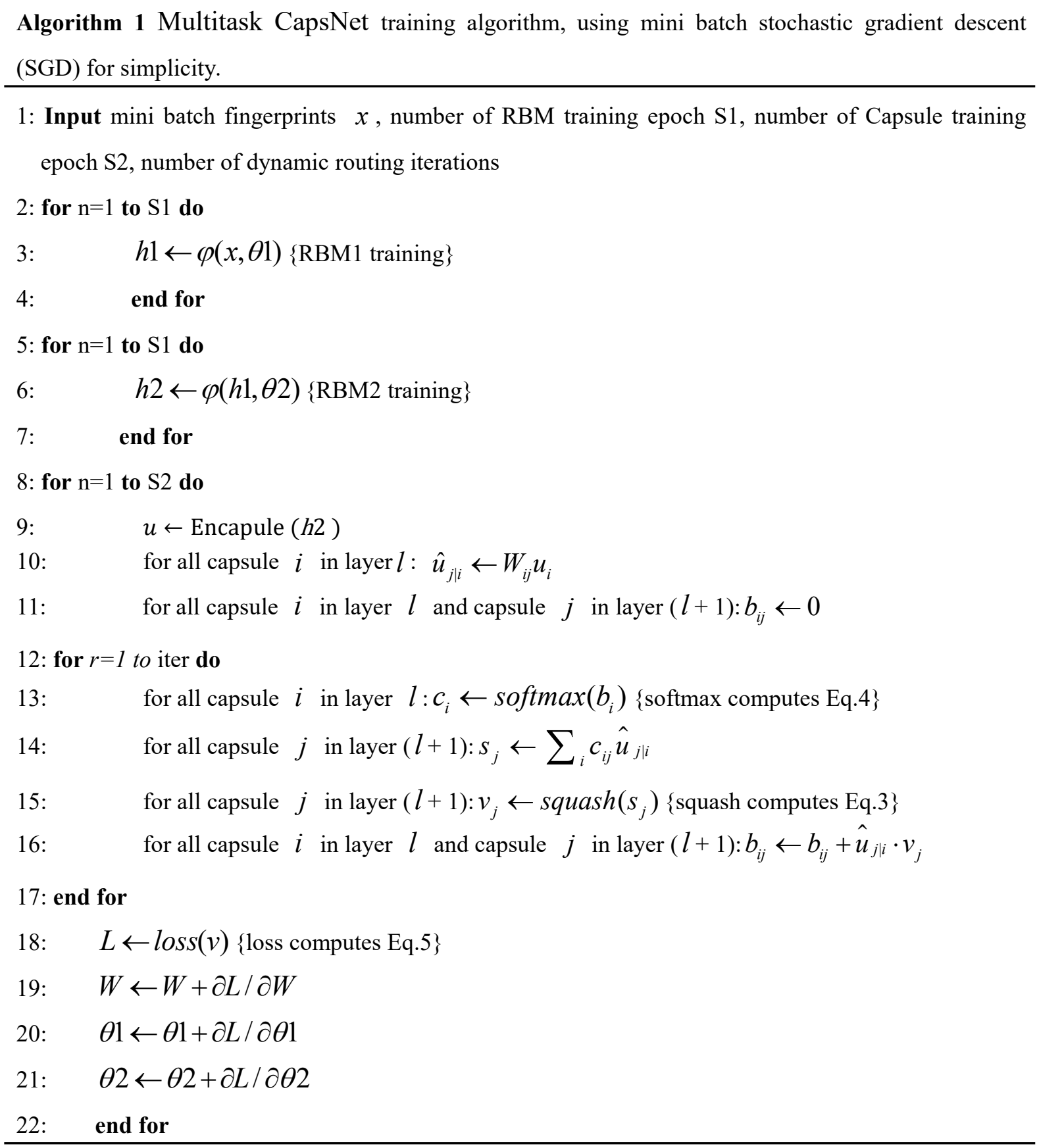

Algorithm 1 summarizes the training procedure of Multitask CapsNet. After training RBM1 and RBM2 layer by layer (line2-7), $h 2$ was packed as capsules $u$ (line 9). $\hat{u}$ denotes the capsule after computing the different contributions of $u$. And then routing algorithm was used to generate digit capsules (line11-17). Lines 18 to 21 are meant to indicate taking a gradient step to update the network parameters. Note that 
$\partial L / \partial W, \partial L / \partial \theta 1$ and $\partial L / \partial \theta 2$ indicate the gradient of contribution matrix, parameters of RBM1 and RBM2 respectively.

\section{S7: Optimal Hyper-parameter Settings}

Table S9. Optimized Hyper-parameter Values of Multitask DBN Model.

\begin{tabular}{cc}
\hline hyper-parameter & setting \\
\hline Numbers of RBM & 2 \\
Number of nodes in the hidden feature layer & 256 \\
Number of nodes in the PrimaryCaps layer & 128 \\
Iteration of RBM & 100 \\
Iteration of network & 200 \\
Learning rate of RBM & 0.001 \\
Learning rate of network & 0.005 \\
Activation & Relu \\
Batch size & 148 \\
Optimizer & Adam \\
\hline
\end{tabular}

Table S10. Optimized Hyper-parameter Values of Multitask CNN Model.

\begin{tabular}{cc}
\hline hyper-parameter & setting \\
\hline Numbers of Convolutional layer & 3 \\
Numbers of Fully connected layer & 2 \\
Kernel_size & 3 \\
Filter & $16 / 32 / 64$ \\
Number of nodes in the Hidden layer & $1024 / 2$ \\
Learning rate of network & 0.001 \\
Activation & Relu \\
Batch size & 148 \\
Optimizer & Adam \\
\hline
\end{tabular}


Table S11. Optimized Hyper-parameter Values of Multitask LSTM Model.

\begin{tabular}{cc}
\hline hyper-parameter & setting \\
\hline Numbers of each input & 8 \\
Numbers of input step & 21 \\
Number of the Hidden layers of LSTM Cell & 1 \\
Number of nodes in the Hidden layer of LSTM Cell & 6 \\
Learning rate of network & 0.05 \\
Activation & Relu/Softmax \\
Batch size & 148 \\
Optimizer & Adam \\
\hline
\end{tabular}

Table S12. Optimized Hyper-parameter Values of Multitask MLP Model.

\begin{tabular}{cc}
\hline hyper-parameter & setting \\
\hline Numbers of Fully connected layer & 2 \\
Number of nodes in the Hidden layer & $256 / 2$ \\
Learning rate of network & 0.001 \\
Activation & Relu/Sotfmax \\
Batch size & 148 \\
Optimizer & Adam \\
\hline
\end{tabular}

